\title{
A Co-optimization Model of Natural Gas Supply and Electric Power Systems
}

\author{
Worawat Nakawiro \\ Department of Electrical Engineering, Faculty of Engineering, \\ King Mongkut's Institute of Technology Ladkrabang, Chalongkrung Road, Ladkrabang, \\ Bangkok 10520, Thailand \\ E-mail: nworawat@gmail.com
}

\begin{abstract}
In Thailand, natural gas has been the primary source of fuel for power generation for the past few decades due to availability of indigenous resources. With continuous load growth and near depletion of domestic natural gas supply, renewable energy will play an increasing role in power industry in a close future. To achieve that, energy storage would become the key enabler. Therefore, this paper proposes a co-optimization steady-state model of a-coupled system of gas supply network and electric power system with integration of an energy storage device namely power-to-gas. The IEEE-14 bus power system coupled with the 20-node and 24 pipeline natural gas system is used to verify effectiveness of the proposed method through computer simulation. The results show the capability in maintaining the system variables within the statutory security limits and reducing power losses. Moreover, the proposed method has another feature that can optimally curtail loads of the two systems when experiencing stressful conditions.
\end{abstract}

Keywords: Co-optimization, integrated energy system, power-to-gas conversion, energy storage, renewable energy.

ENGINEERING JOURNAL Volume 23 Issue 2

Received 28 August 2018

Accepted 9 January 2019

Published 31 March 2019

Online at http://www.engj.org/

DOI:10.4186/ej.2019.23.2.135 


\section{Introduction}

Electricity production from large-scale fossil-fired power plants have been the cost-effective solution to electrify consumers. This trend has significantly been affected by the declined cost of distributed generation technologies. Therefore, we can observe an increasing number of renewable energy (RE) source connections to the power grid. In Thailand, the latest power development plan (PDP) 2015-2036 indicates the plan to increase share of $\mathrm{RE}$ in power generation which reducing the reliance of natural gas-fired power generation from $64 \%$ of the total installed capacity now to $30-40 \%$ [1]. This plan was proposed to cope with the projection that the national gas reserve capacity will be depleted in a very near future.

It was well known that energy produced from $\mathrm{RE}$ is intermittent due to weather conditions. With varying demand, residual energy is fluctuating in positive and negative directions. This fact gives both opportunities and threats to the power system. If this residual is not well managed, it would cause technical issues to the system operation such as overvoltage, increase losses or misoperation of protective devices, etc. The advantage of excess energy is that it can be stored for future uses. Hence, energy storage is the key enabler to achieve high penetration of RE. Power-to-gas (P2G) is the technology that uses excess electricity to produce hydrogen $\left(\mathrm{H}_{2}\right)$ in the electrolysis process [2]. The produced $\mathrm{H}_{2}$ converted to methane and thereby produce synthetic natural gas (SNG) which can thereby either used or stored in the gas network. Alternatively, it can be used to support mobility applications as well. Moreover the P2G conversion process can generate heat either from the fuel cell or from the methane conversion process [3]. The selection of energy storage depends on economic costs and technical implications. The impact of three types of energy storage namely P2Gm pumped hydro storage and compressed air energy storage on a power system with different penetration level of wind energy were investigated in [4]. In France, there was a proposal to apply $20 \mathrm{GW}$ P2G facility to convert the surplus power during off-peak period to hydrogen such that the nuclear power plants need not to be shut down [6]. However practical implementation of P2G is still limited mainly in Germany [7] and other European countries [8]-[9].

Several previous works dealing with analysis the coupled gas and electricity network. Some representative works in [10]-[14] are discussed here. A detailed nonlinear model of gas and power systems was proposed in $[10]$ to find a steady state operating point of the coupled system. However, optimal solution was not investigated in this work. The impact of natural gas infrastructure such as pipeline outage on cost and operation of electric power system was presented in [11] in which a security constrained unit commitment was developed. The so-called integrated energy system (IES) model was developed in [12] to study economic efficiency of multiple energy sources including coal and natural gas supply to the electrical load by solving a linear programming problem. The so-called co-optimization planning process that optimizes two or more related energy resources was proposed in [13]. A dynamic model describing interaction between the natural gas supply network fueling micro-turbines was developed in [14] and a simulation technique for two subsystems with different time scales was also proposed.

With integration of P2G, a steady state model of the coupled gas supply and power system was presented in [15]. Variation of wind power and load was applied to study the impact of P2G in mitigating the impact on both subsystems. A proposal of storing renewables in the gas network to support seasonal load variation in UK was presented in [16] the simulation of which relies on the framework presented in [17] by the same authors. A day-ahead scheduling of P2G and gas load forecasting were proposed in [18]. The synthesis gas is optimally used in normal operation under the competitive environment. Uncertainty of wind power is incorporated in an optimization model using interval probability theory in [19].

Based on the above literature review, the optimization problem of most of the previous works is linear model using DC power flow in determining state of the power system. It is well known that linear optimization problems can be easily solved within relatively fast computing time. However, some engineering issues such as reactive power are omitted due to linear approximation. This paper attempts to fill this gas by presenting a co-optimization nonlinear model allowing detailed analysis of the coupled electric power and gas supply systems. Moreover the computing effort for solving the proposed model should be acceptable. The rest of this paper is organized as follows. The model of integrated system is described in section 2 . The co-optimization problem is formulated where all constraints and variables are listed in section 3 . The test system is given in section 4. Simulation results are explained in section 5. Finally the paper is concluded and future research outlook is discussed. 


\section{Modeling the Integrated Energy System}

The integrated energy system consists of two sub systems namely gas supply network and electric power system. The model of each component in the gas sub system is explained in section 2.1 whereby the set of gas network equations are discussed in section 2.2. The power system model consisting of power flow equations and other operational limits are given in section 2.3.

\subsection{Natural Gas Network Component}

\subsubsection{Gas well}

Natural gas is injected to the gas network from gas wells. The gas injection available at gas well $i$ each time $t$ period $g_{s i, t}$ is restricted by

$$
g_{s i, \min } \leq g_{s i, t} \leq g_{s i, \max } \forall i \in \mathbf{S}_{\mathbf{G W}}
$$

where $g_{s i \text { min }}$ and $g_{s i \text { max }}$ are the minimum and maximum supply of gas well $i$ and $\mathbf{S}_{\mathbf{G W}}$ is the set of all gas wells.

\subsubsection{Gas pipeline}

Gas flows through two types of pipelines namely passive and active pipelines. The pressure difference between any two nodes drives the flow in passive branches while external power is used to direct the gas flow in active branches. The gas flows from node $i$ to node $j$ for passive branches is expressed by

$$
f_{i j}=S_{i j} C_{i j} \sqrt{p_{i}^{2}-p_{j}^{2}}, \forall(i, j) \in \mathbf{B}_{\mathbf{P}}
$$

where $p_{i}$ and $p_{j}$ are the gas pressure at nodes $i$ and $j$ respectively; $C_{i j}$ is the constant describing physical characteristics of each pipeline; $\mathrm{B}_{\mathrm{P}}$ is the set of containing the two end points of all passive pipelines; $S_{i j}$ is the sign function to indicate the flow direction given by

$$
S_{i j}=\left\{\begin{array}{cc}
1 & ; p_{i}>p_{j} \\
-1 & \text { otherwise }
\end{array}\right.
$$

For an active pipeline, the gas can flow from a lower-pressure node $i$ to a higher-pressure node $j$ due to operation of the compressor $k$ according to

$$
f_{k, i j} \geq S_{i j} C_{i j} \sqrt{p_{i}^{2}-p_{j}^{2}}, \forall(i, j) \in \mathbf{B}_{\mathbf{A}}
$$

where $\mathbf{B}_{\mathbf{A}}$ is the set of containing the two end points of all active pipelines.

\subsubsection{Compressor}

Gas compressor stations are installed to compensate the gas flow loss during the transportation due to frictional resistance. An empirical study under ideal gas assumption shows that the horsepower consumption required by the compressor $k$ installed between the suction node $i$ and the discharge node $j$ can be calculated by [20]. 


$$
H_{k, i j}=B_{k} f_{k, i j}\left[\left(\frac{p_{j}}{p_{i}}\right)^{Z_{k i}\left(\frac{\alpha-1}{\alpha}\right)}\right]
$$

where $B_{k}=\frac{3554.58 T_{k i}}{\eta_{k}}\left(\frac{\alpha}{\alpha-1}\right) ; \eta_{k}$ is the compressor efficiency; $\alpha$ is specific heat ratio; $T_{k i}$ is the compressor suction temperature; $\mathrm{f}_{\mathrm{k}}$ is the flow rate through the compressor and $Z_{k i}$ is the gas compressibility factor at the compressor inlet.

The amount of gas tapped from the network for driving the compressor station $k$ is computed by

$$
\tau_{k}=\alpha_{k}+\beta_{k} H_{k, i j}+\gamma_{k} H_{k, i j}^{2}
$$

where $\alpha_{k}, \beta_{k}$ and $\gamma_{k}$ are the coefficient of the quadratic approximation. The compression ratio at the compressor $k$ is defined by the ratio of the discharge node to the suction node as

$$
R_{k}=\frac{p_{j}}{p_{i}}
$$

It is restricted by the following constraint

$$
R_{k, \min } \leq R_{k} \leq R_{k, \max }
$$

where $\mathrm{R}_{\mathrm{k}, \text { min }}$ and $\mathrm{R}_{\mathrm{k}, \max }$ are minimum and maximum allowable compression ratio of the compressor $k$

\subsubsection{Gas storage}

Gas storage is the facility used to coordinate the gas flow in multi-period operation. The gas flow (in/out) the storage at time $t$ denoted by $g_{f, t}$ is constrained by

$$
-g_{\text {lev }, t} \leq g_{f, t} \leq \min \left(g_{\text {ava }, t}, g_{\text {cap }}-g_{\text {lev }, t}\right) \forall t \in T
$$

where $g_{l e v, t}$ is the gas level in the storage at time $t, g_{\text {ava, } t}$ is the available amount of gas produced by P2G and can be stored at time $t, g_{\text {cap }}$ is capacity of the gas storage and $T$ is the set of all time steps. The negative sign of $g_{f, t}$ indicates the outflow from the storage to the network whereas the positive sign shows the gas flowing into the storage. It is noted that the maximum gas outflow is the gas level in the storage tank at that time step whereby the maximum gas inflow is limited by the remaining capacity of the storage tank at that time step.

\subsubsection{Gas load}

In the gas network, loads are categorized in two groups namely; gas loads such as residential and industrial consumers and electric generators. For gas-fired generators, heat rate (HR) is defined by the ratio of thermal energy consumed to electrical energy produced in MJ/MWh. Efficiency of generator $\eta_{G}$ is a unitless ratio of electrical energy output to the thermal energy input. Therefore the efficiency can be written as [15] 


$$
\eta_{G}=\frac{3600}{H R}
$$

Low heating value (LHV) of natural gas is defined by the thermal energy provided by a given unit of natural gas. Typically LHV is in the range between $35.4-39.12 \mathrm{MJ} / \mathrm{m}^{3}$. This value varies depending on the quality of gas. Therefore the amount of gas required by generator $G$ to produce electrical power $P_{g}$ is expressed by

$$
g_{G}=\frac{H R \times P_{g}}{L H V}=\left(\frac{3600}{\eta_{g} L H V}\right) P_{g}
$$

The average value of $\mathrm{LHV}$ of $37.26 \mathrm{MJ} / \mathrm{m}^{3}$ is used in this paper.

\subsubsection{Power-to-gas conversion}

The electrical power from renewable sources in excess from the demand can be used to produce hydrogen by the electrolysis process described by

$$
2 \mathrm{H}_{2} \mathrm{O} \rightarrow 2 \mathrm{H}_{2}+\mathrm{O}_{2}
$$

Then the hydrogen is used to produce methane in the so-called methanization process according to

$$
\mathrm{CO}_{2}+4 \mathrm{H}_{2} \rightarrow \mathrm{CH}_{4}+2 \mathrm{H}_{2} \mathrm{O}
$$

In this paper, efficiency of P2G conversion $\eta_{P 2 G}$ is defined by the ratio between energy density of the produced gas and the electrical power consumed by the P2G given by [15]

$$
\eta_{P 2 G}=\frac{\frac{g_{P 2 G} L H V}{3600}}{P_{P 2 G}}
$$

where $g_{P 2 G}$ is the gas to be produced by P2G conversion and $P_{P 2 G}$ is the electrical power required for the $\mathrm{P} 2 \mathrm{G}$ conversion.

\subsection{Gas Network Model}

For a given set of demand and supply rates and operation of compressors, the gas flow is governed by conservation of mass balance at each node which can be written as [20]

$$
(\mathbf{A}+\mathbf{U})\left[\begin{array}{c}
\mathbf{f}\left(p_{i}, p_{j}\right) \\
\mathbf{f}_{c}
\end{array}\right]+\left[\begin{array}{c}
\left(1-x_{g l}\right) \mathbf{g}_{L} \\
\mathbf{g}_{G}
\end{array}\right]+\mathbf{C} \boldsymbol{\tau}=0
$$

where $\mathbf{A}$ is the signed matrix representing network interconnection whose elements correspond to node $i$ and node $j$; the $\mathbf{U}$ matrix representing connection of compressors to nodes; $\mathbf{f}\left(p_{i}, p_{j}\right)$ is the vector of gas flows through passive pipelines; $\mathbf{f}_{c}$ is the vector of gas flow through compressors (active pipelines); $\mathbf{g}_{L}$ and $\mathbf{g}_{G}$ are the vectors of gas load consumption and gas required by power generating units, respectively; $x_{g l}$ is 
the ratio of the gas load to be curtailed; $\mathbf{C}$ is the matrix representing the nodes from which the gas for compressor is tapped; $\tau$ is the vector of amount of gas required for all compressors.

\subsection{Electric Power System Model}

The set of nonlinear active and reactive power flow equations are used in this paper. We have introduced extra variables indicating curtailment factors of renewable power infeed and power demand to ensure feasibility of the solution.

\subsubsection{Power mismatch}

Power mismatch at each bus is defined by the difference between the total power generation from both thermal and renewable sources and the sum of power demand and the total power flowing out of that bus. In this paper, active power mismatch at bus $i$ is described by:

$$
\begin{gathered}
\Delta P_{i}=P_{G i}+x_{w} P_{W i}+x_{s} P_{S i}-\left(1-x_{p l}\right) P_{D i} \\
-\sum_{i=1}^{n} V_{i} V_{j} Y_{i j} \cos \left(\delta_{i}-\delta_{j}-\theta_{i j}\right)=0
\end{gathered}
$$

where $P_{G i}, P_{W i}$ and $P_{S i}$ are active power generated from thermal unit, wind and solar farms at bus $i$,respectively; $x_{w}$ and $x_{s}$ are the ratio of wind and solar power to be injected to the power system, respectively; $x_{p l}$ is the ratio of electric power load to be curtailed at bus $i$ and $P_{D i}$ is the active power demand at bus $i$. The complex element $i j$ of bus admittance matrix is written as $Y_{i j} \angle \theta_{i j}$. The voltage phasor of bus $i$ is denoted by $V_{i} \angle \delta_{i}$.

It is assumed in this study that wind and solar farms maintain unity power factor at the point of common coupling (PCC) and power factor of the load remains unchanged in case of curtailment. Therefore, reactive power mismatch at bus $i$ is described by

$$
\begin{aligned}
\Delta Q_{i} & =Q_{G i}-\left(1-x_{p l}\right) Q_{D i} \\
& -\sum_{i=1}^{n} V_{i} V_{j} Y_{i j} \sin \left(\delta_{i}-\delta_{j}-\theta_{i j}\right)=0
\end{aligned}
$$

where $Q_{G i}$ is the reactive power generated from thermal unit at bus $i$ and $Q_{D i}$ is the reactive power demand at bus $i$.

\subsubsection{Power generation limits}

The active power output of thermal generating units is maintained within their limits

$$
P_{G i, t, \min } \leq P_{G i, t} \leq P_{G i, t, \max }, \forall i \in \mathbf{S}_{\mathbf{G}} \forall t \in T
$$

where $\mathbf{S}_{\mathbf{G}}$ is the set of thermal generating units and $P_{G i, t, \text { min }}$ and $P_{G i, t, \text { max }}$ are minimum and maximum limits of power generated from unit $i$ at time $t$ governed by ramp limits given by

$$
\begin{aligned}
& P_{G i, t \text { min }}=\max \left(P_{G i, \text { min }}, P_{G i, t-1}-D R_{i}\right) \\
& P_{G i, t, \text { max }}=\min \left(P_{G i, \text { max }}, P_{G i, t-1}+U R_{i}\right)
\end{aligned}
$$


where $D R_{i}$ and $U R_{i}$ are down-and up- ramp limits of generating unit $i$. and $P_{G i \text {,min }}$ and $P_{G i \text {,max }}$ are minimum and maximum generation capacity of the unit $i$, respectively.

As a dependent variable, reactive power output of generating unit $i$ is maintained within

$$
Q_{G i, \min } \leq Q_{G i, t} \leq Q_{G i, \max }, \forall i \in \mathbf{S}_{\mathbf{G}} \forall t \in T
$$

where $Q_{G i, \min }$ and $Q_{G i, \max }$ are minimum and maximum limits of reactive power of unit $i$ at time $t$.

\subsubsection{Voltage limits}

For all load buses, voltage magnitude is maintained within the allowable limit

$$
V_{i, \text { min }} \leq V_{i, t} \leq V_{i, \text { max }}, \forall i \in \mathbf{S}_{\mathbf{L B}} \forall t \in T
$$

where $V_{i, \min }$ and $V_{i, \max }$ are minimum and maximum limits of voltage magnitude at bus $i$ and $\mathbf{S}_{\mathbf{L B}}$ is the set of load buses. It is assumed that load bus voltages can vary within $\pm 5 \%$ of the nominal value.

\subsubsection{Power flow limits}

Power flow over each transmission line in the power system is restricted by the maximum capacity given by

$$
\left|S_{L, i j}\right| \leq S_{L, i j, c a p}
$$

where $\left|S_{L, i j}\right|$ is the apparent power flow over line connected between bus $i$ and bus $j$ and $S_{L, i j, c a p}$ is the MVA capacity of line connected between bus $i$ and bus $j$

\section{Problem Formulation}

A nonlinear co-optimization problem for daily operation of integrated gas supply and electric power system with P2G storage is sequentially solved for each time step (i.e. 1 hour as in this paper). The objective is to minimize the combined function

$$
\begin{aligned}
f= & \sum_{i=1}^{N_{G}} f_{c}^{\prime}\left(P_{G i}\right)+\sum_{j=1}^{M} f_{g}^{\prime}\left(g_{s j}\right)+g_{P 2 G}^{\prime} \\
& +\left(1-x_{w}\right)+\left(1-x_{s}\right)+k\left(x_{p l}+x_{g l}\right)
\end{aligned}
$$

where $N_{G}$ is the number of thermal generating units and $M$ is the number of natural gas sources. The normalized fuel cost $f_{c}^{\prime}\left(P_{G i}\right)$ of thermal generating units can be determined from

$$
f_{c}^{\prime}\left(P_{G i}\right)=\frac{f_{c}\left(P_{G i}\right)-f_{c}\left(P_{G i, \min }\right)}{f_{c}\left(P_{G i, \max }\right)-f_{c}\left(P_{G i, \min }\right)}
$$

The fuel cost of generating unit $i$ given by

$$
f_{c}\left(P_{G i}\right)=a_{i} P_{G i}^{2}+b_{i} P_{G i}+c_{i}
$$


where $a_{i}, b_{i}$ and $c_{i}$ are coefficients of fuel cost function of unit $i$. Similarly, the gas supply cost of source $j$ expressed by $f_{g}^{\prime}\left(g_{s i}\right)$ is also normalized in $[0,1]$. The usage of gas produced by P2G is also normalized with respect to the storage tank capacity

$$
g_{P 2 G}^{\prime}=\frac{\left(g_{c a p}-x_{P 2 G} g_{l e v, t}\right)}{g_{c a p}}
$$

where $x_{P 2 G}$ represents the percentage of $\mathrm{P} 2 \mathrm{G}$ produced gas drawn out from the storage and supplied to the gas network.

The next two terms of (23) intends to maximize utilization of renewable sources by minimizing the percentage of wind and solar power curtailments. In some infeasible cases, the additional objective to minimize the total electricity and gas load shedding will be introduced $(k=1)$. This is to ensure that a feasible solution can always be found.

In this study, the set of equality constraints consists of the power mismatch equations (16)-(17), the gas network model (15), the compressor power consumption (5), the gas consumed by compressor (6) and the compression ratio (7). The set of inequality constraints consists of reactive power limit (20) and line power flow limit (22).

The vector of decision variables to be determined can be expressed by

$$
\mathbf{X}=\left[\begin{array}{lll}
\mathbf{x}_{\mathrm{p}} & \mathbf{x}_{\mathrm{g}} & \mathbf{x}_{\mathbf{c}}
\end{array}\right]
$$

where $\mathbf{x}_{\mathbf{p}}$ is the vector of power system variables as

$$
\mathbf{x}_{\mathbf{p}}=\left[\delta_{1}, \ldots, \delta_{N}, V_{1}, \ldots, V_{N L}, P_{G, 1}, \ldots, \ldots, P_{G, N_{G}}\right]
$$

where $N$ is the number of buses. The vector of gas network variables is

$$
\mathbf{x}_{\mathbf{g}}=\left[g_{s, 1}, \ldots, g_{s, N_{G W}}, p_{N_{G W+1}}, \ldots, p_{N_{N}}, f_{1}, \ldots, f_{N_{c}}, H_{1}, \ldots, H_{N_{c}}, \tau_{1}, \ldots, \tau_{N_{c}}\right]
$$

where $N_{G W}$ is the number of gas wells, $N_{N}$ is the number of nodes in the gas network and $N_{C}$ is the number of compressors. Finally the vector of variables used to control feasibility of the solution is

$$
\mathbf{x}_{\mathbf{c}}=\left[x_{w}, x_{s}, x_{p l}, x_{g l}\right]
$$

Note that any optimization package can be applied to solve the proposed problem. Moreover, any nonlinear equation solver such as Newton Raphson is not need.

\section{Test System and Simulation Setting}

The effectiveness of the developed method is evaluated on a test system namely IEEE-30 bus power system with 15-node natural gas network as shown in Fig. 1. In the power system, two gas-fired generating units are located at bus 1 and bus 2 . There are electrical loads located at 24 buses throughout the network. Wind farm is connected at bus 30 whereby solar PV is connected at bus 22 . In the gas network, two gas wells are at node 1 and 2. Gas loads are located at nodes 3, 13, and 14. Gas-fired generators G1 and G2 receive gas from nodes 4 and 15 of the gas supply network, respectively. Four compressors are installed on the pipelines to boost up gas pressure and use natural gas from the network as the fuel. A gas storage receives SNG produced by P2G and discharges to the network at node 13 . 


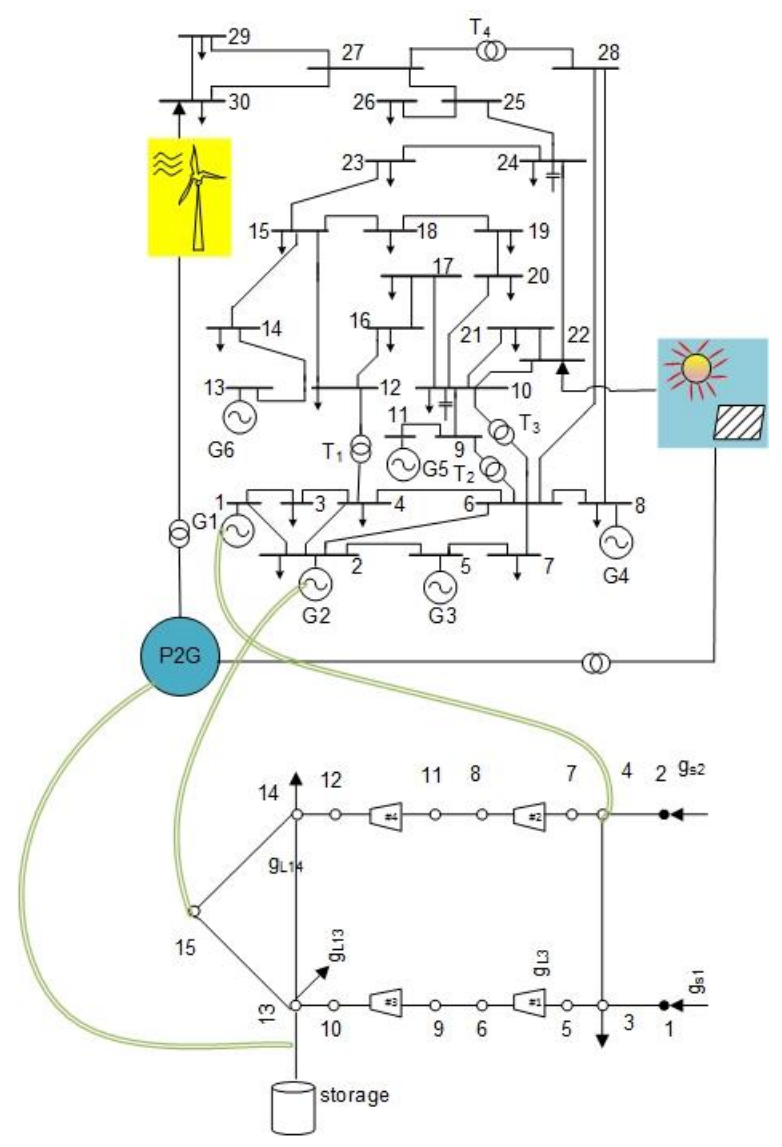

Fig. 1. The test system.

The daily profile of electricity and gas loads is shown in Fig. 2. The daily profile of wind and solar power is shown in Fig. 3. Note that both profiles are given in a normalized multiplicative factor at each hour of the day. In this paper, it is assumed that the maximum capacity of electricity load is $200 \mathrm{MW}$ and the maximum capacity of gas load is $14.09 \mathrm{Mft}^{3}$. There are two peaks of electricity load occurring at 12 and 19 hours (with the multiplicative factor of 0.9909 ) whereby the peak of gas load occurs at 20 hour hours (with the multiplicative factor of 0.8897 ) (see Fig. 2). Then, the total peak electricity load is $99.09 \%$ of $200 \mathrm{MW}$ which is $198.18 \mathrm{MW}$ whereby the total peak gas load is $88.97 \%$ of $14.09 \mathrm{Mft}^{3}$ which is $12.5359 \mathrm{Mft}^{3}$. The wind and solar capacities are 30 and $20 \mathrm{MW}$, respectively. The wind and solar power at each time step of the day is defined as the percentage (ratio as in this paper) of the capacity in a similar manner to the load profiles explained earlier.

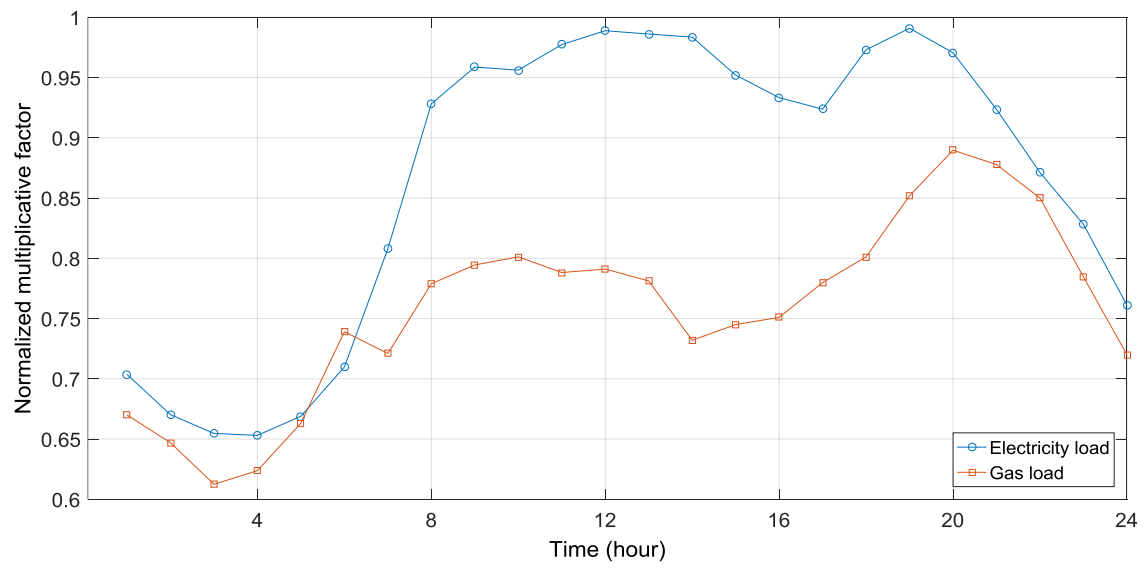

Fig. 2. Daily profile of electricity and gas loads. 


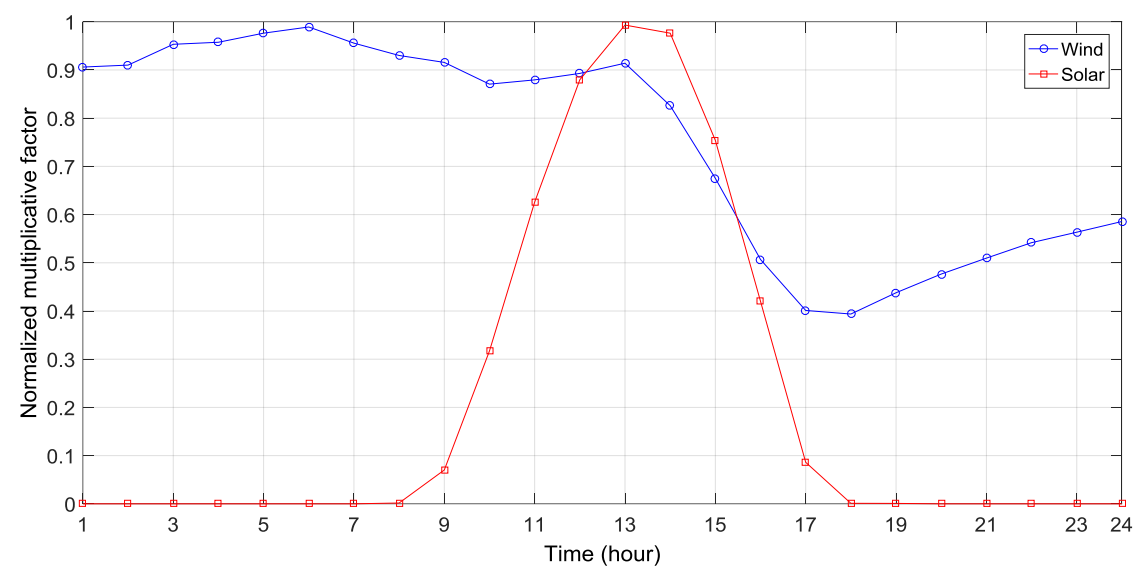

Fig. 3. Daily profile of wind and solar.

The simulations were carried out in MATLAB M-files and the nonlinear constrained optimization routine 'fmincon' with 'active-set' algorithm [21] was used to find the optimal operating point of both subsystems. Simulations in this paper were carried out on a computer laptop with Intel core i5-3317 U 1.7 GHz, Windows 10, 12 GB RAM. The total CPU time used for solving 24 time steps is $108.84 \mathrm{~s}$ or $4.53 \mathrm{~s}$ for each time step on average.

\section{Simulation Results}

As mentioned earlier, this paper proposes a nonlinear model that co-optimizes operation of electricity and gas supply systems with an energy storage facility namely power-to-gas. The simulation studies to be presented in this section are aimed at demonstrating improvement of the system performance both in normal and abnormal operation due to the proposed method. The results are compared with the case that the proposed method was not implemented. Comparison with other control strategies is beyond the scope of this paper.

Figure 4 shows the impact of different RE levels to the amount of SNG to be stored in the gas storage. In this simulation, SNG is intended to be used to offset the day- and night peaks of the gas load during 9-13 and 18-22 hours, respectively. It is logical that the SNG produced at $120 \%$ of nominal RE is the highest. Therefore the SNG can help reduce both day and night peaks. Moreover, it can be seen that SNG built up during 1-6 hours due to high wind power and low load. It is assumed in this simulation that the storage tank capacity is $0.2 \mathrm{Mft}^{3}$. At the $120 \% \mathrm{RE}$ level, the SNG filled up the capacity during 7-9 hours and later released to help offset the gas load. It should be noted that SNG does not increase much during the day time because of very limited surplus power. The SNG is released from the storage again during the peak (19-21) hours.

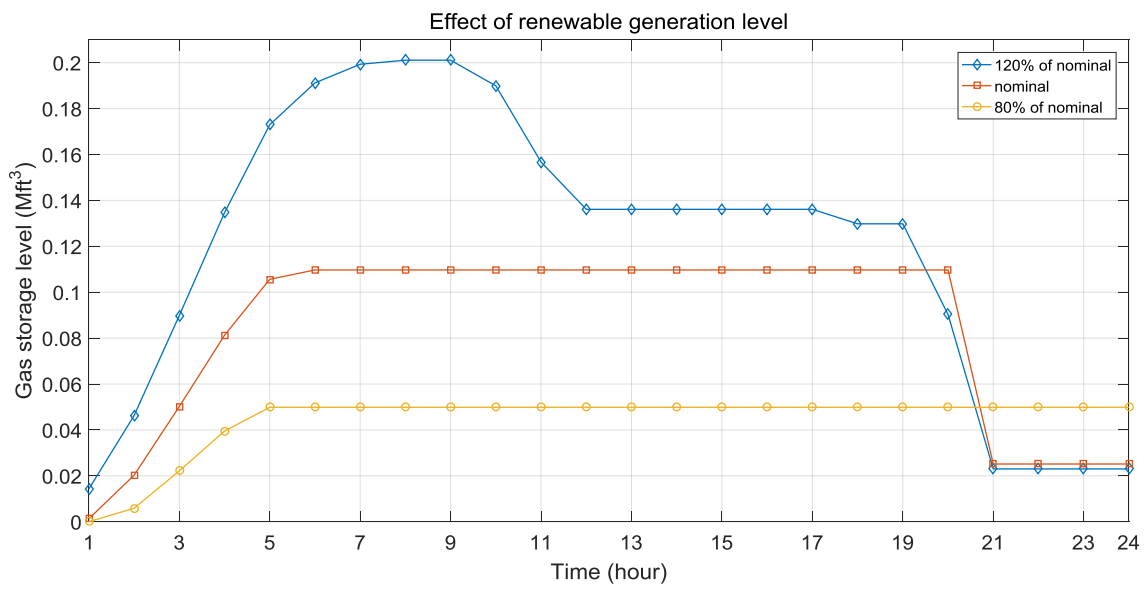

Fig. 4. Effect of RE levels on the gas storage. 
Figure 5 shows the voltage profiles at the wind point of common coupling (PCC) at bus 30 . Without the proposed optimization, the voltage level increases during this 5-hour period due to excessive power injected to the power grid. This paper adopts the grid code of the National Grid Company (NGC) of United Kingdom in which the power plant must be able to provide full reactive power support at voltage $\pm 5 \%$ around the nominal value [22]. Therefore, if the proposed method is not used the wind PCC bus voltage violates the upper bound of the voltage limit due to excessive wind availability during the night time. With the proposed method, this surplus power is fed to P2G to produce SNG as seen from the reduced voltage level below the security limit as well.

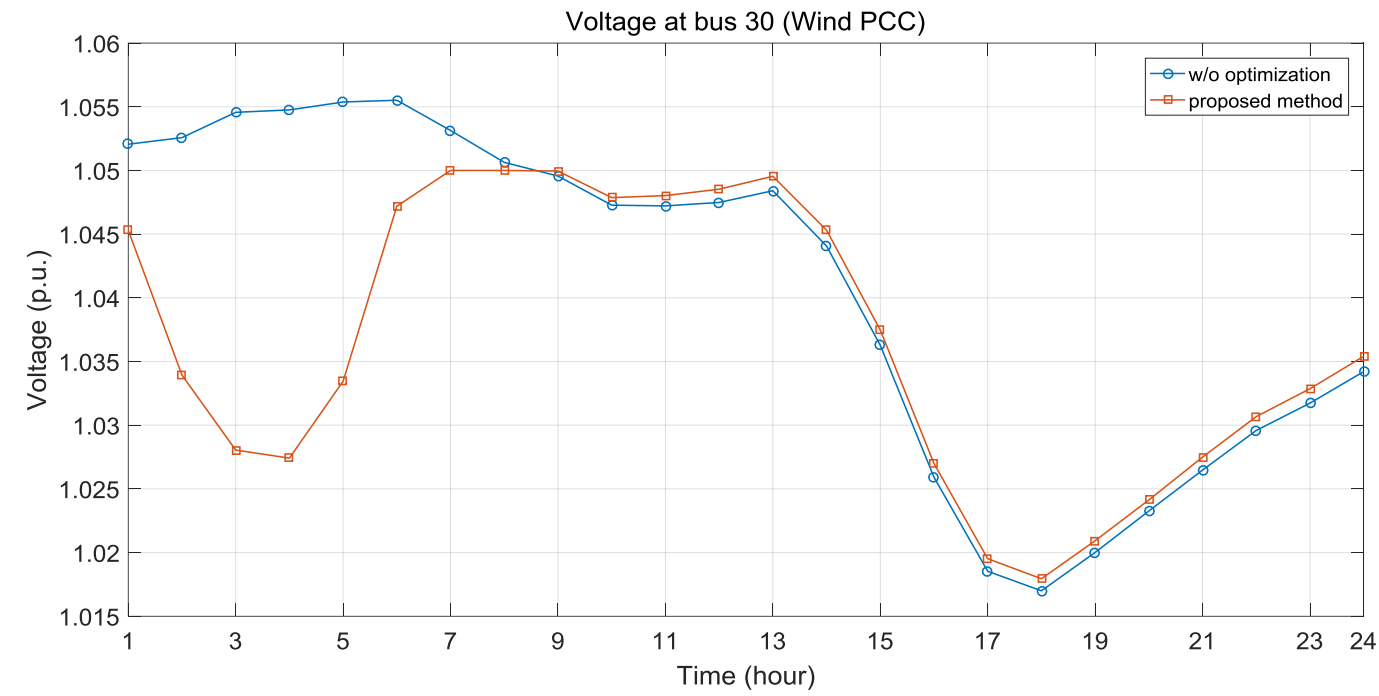

Fig. 5. Wind PCC bus voltage.

The voltage profiles at the PCC of solar farm are shown in Fig. 6. It can be seen that without the proposed optimization, voltage fluctuates in a pattern similar to the solar irradiance. With the proposed optimization method, voltage fluctuation during daytime is less and the level is higher than the case without optimization. This would help flatten voltage level across the system and thereby help reduce losses.

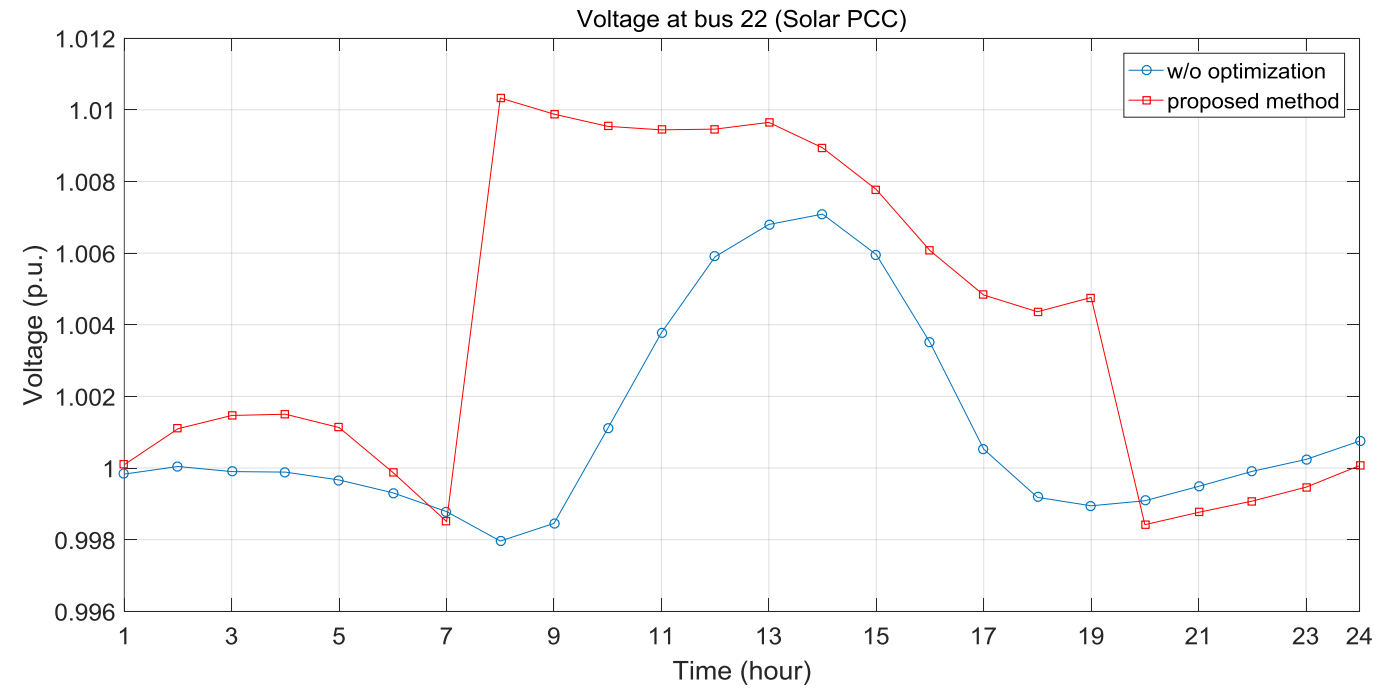

Fig. 6. Solar PCC bus voltage.

Figure 7 shows comparison of power losses in cases of with and without optimization. It is noted that the proposed method can optimally manage the integrated system and thereby resulting in reduced losses at 
every time step in the day. Loss reduction during hours 1-7 is very significant due to power used by P2G for the conversion process.

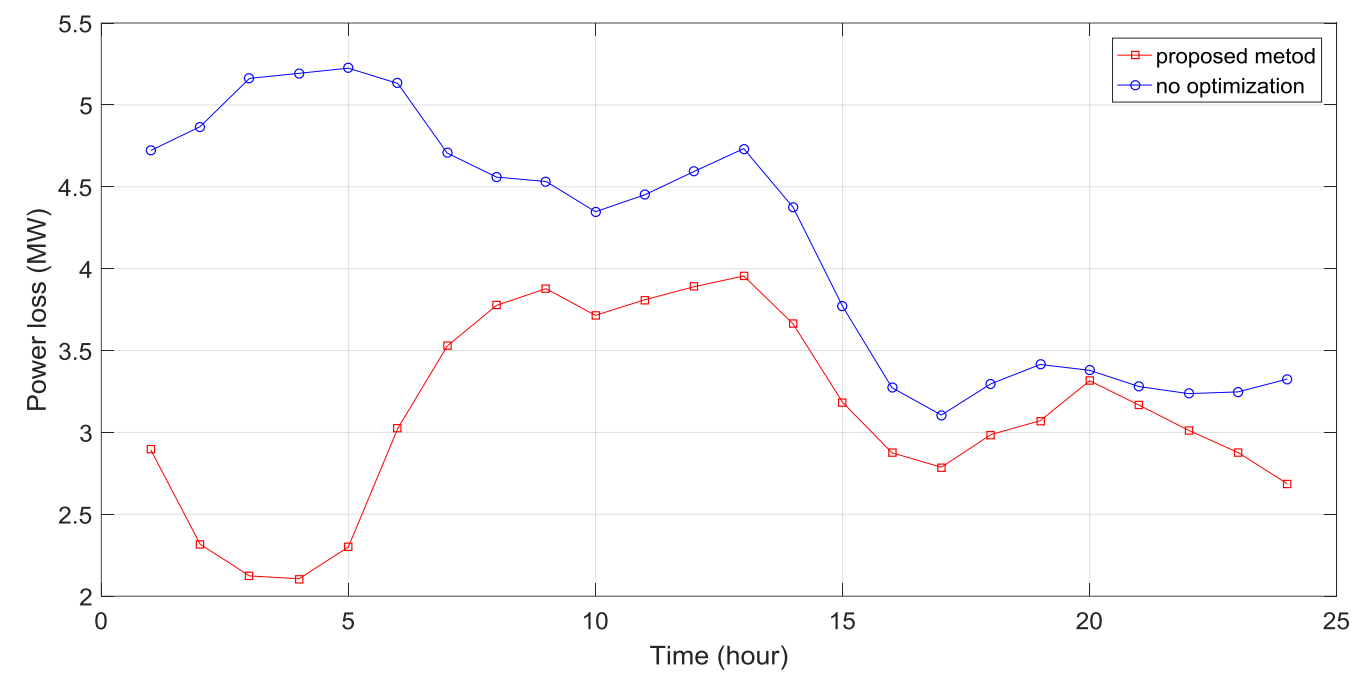

Fig. 7. Power losses.

The other distinct feature of the proposed method is that it can also handle infeasible operating cases. In this simulation, we assumed that there is no power infeed from RE and the gas supply limit is reduced by $20 \%$. This scenario represent low electricity and gas supply while the load remains constant. Therefore, there should be some time periods of the day that the reduced power supply is insufficient to supply the load. To maintain secure and stable operation of the integrated system, optimal load shedding is used. The total electricity generation patterns of the cases with normal and reduced supply are shown in Fig. 8. It is obvious that when there is no RE power infeed, the thermal generating units have to increase their generation which thereby uses more gas. With the reduced gas supply, the operating points at 19-20 hours become infeasible. During this period, the proposed method determines the optimal curtailment of both gas and electrical loads such that the nearest feasible operating point is found.

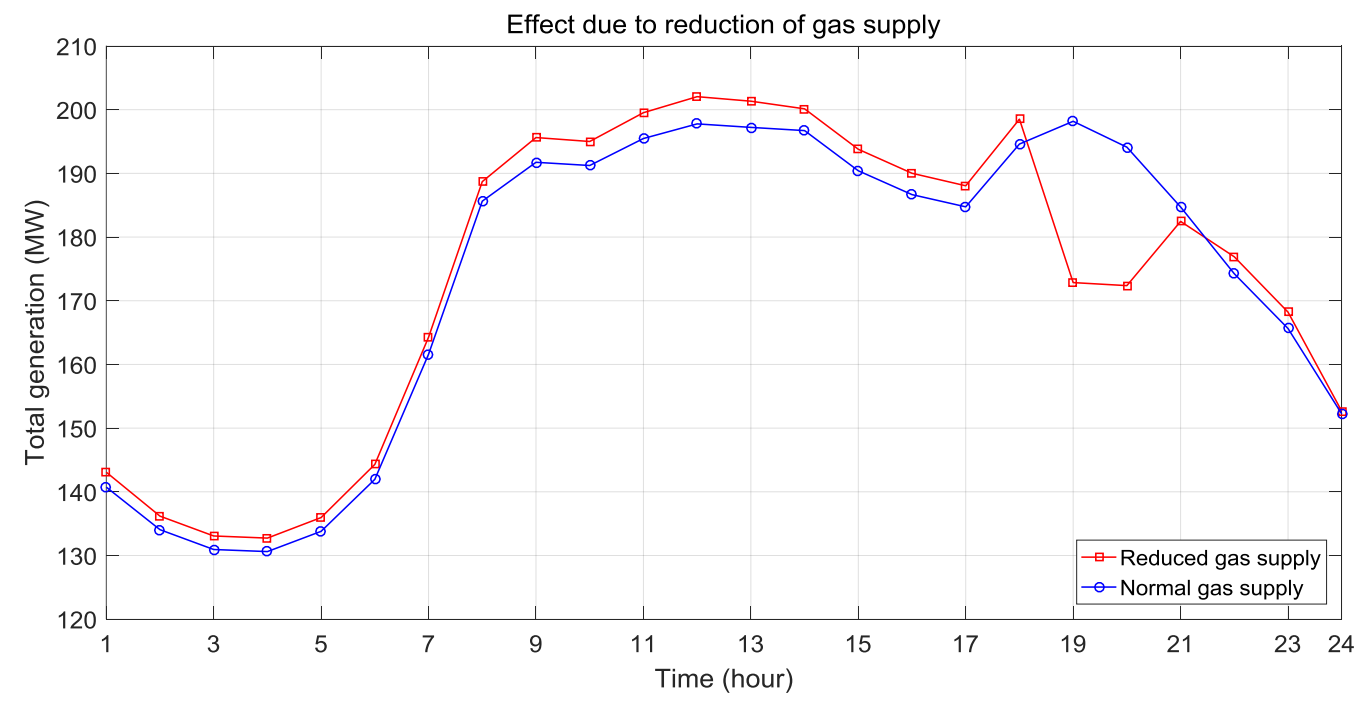

Fig. 8. Effect due to reduced gas supply (without RE). 


\section{Conclusion and Future Outlook}

This paper presents a co-optimization model of natural gas supply and electric power systems with powerto-gas conversion process. The model is formulated as a set of nonlinear equations and solved by the active set algorithm of MATLAB optimization toolbox. However, any other algorithms such as heuristic methods can be applied. Simulation results demonstrate capability of the proposed method in storing surplus power from renewable sources in synthesis gas. Consequently, power required by the conversion process can help reduce power losses especially during nighttime in which wind power is high. Moreover, the proposed method can restore infeasible operating conditions by minimizing load curtailment in both energy systems. It is very well known that there are several uncertainties related to the two systems such as power output from renewable sources and load. These uncertainties can be further incorporated in the proposed model. To handle the problem with uncertainties, the optimization algorithm and implementation procedure may need to be changed. Moreover, power system operation under stressed conditions would experience some type of stability issues such as transient stability. These aspects will be investigated in the future work.

\section{References}

[1] EPPO. (2015). Thailand Power Development Plan 2015 (PDP-2015) [Online]. Available: http://www2.eppo.go.th/power/PDP2015/PDP2015.pdf

[2] U. Bünger, H. Landinger, E. Pschorr-Schoberer, P. Schmidt, W. Weindorf, J. Jöhrens, U. Lambrecht, K. Naumann, and A. Lischke, "Power-to-Gas (PtG) in transportation status quo and perspectives for development," Federal Ministry of Transport and Digital Infrastructure (BMVI), Germany, 2014. Available: http://www.lbst.de/ressources/docs2014/mks-studie-ptg-transport-status-quo-andperspectives-for-development.pdf

[3] M. Lehner, R. Tichler, H. Steinmüller, and M. Koppe, "The power-to-gas concept," in Power to-Gas: Technology and Business Models. Springer, ch. 2, pp. 7-17.

[4] H. S. de Boer, L. Grond, H. Moll, and R. Benders, "The application of power-to-gas, pumped hydro storage and compressed air energy storage in an electricity system at different wind power penetration levels," Energy, vol. 72, pp. 360-370, 2014.

[5] B. Gillessen, K. U. Heinrichs, P. Stenzel, and J. Linssen "Hybridization strategies of power-to-gas systems and battery storage using renewable energy," Int. Journal of Hydrogen Energy, vol. 42 pp. 13554$13567,2017$.

[6] D. Scamman and M. Newborough, "Using surplus nuclear power for hydrogen mobility and power-togas in France," Int. Jour of Hydrogen Energy, vol. 41, pp. 10080-10089, 2015.

[7] S. Schiebahn, T. Grube, M. Robinius, V. Tietze, B. Kumar, and D. Stolten, "Power to gas: Technological overview, systems analysis and economic assessment for a case study in Germany," Int. Journal of Hydrogen Energy, vol. 40 pp. 4285-4294, 2015.

[8] E. Pursuheimo, H. Holttinen, and T. Koljonen, "Path toward $100 \%$ renewable energy future and feasibility of power-to-gas technology in Nordic countries," IET Renew. Power Gener, vol. 11, no. 13, pp. 1695-1706, 2017.

[9] V. Heinisch, "Modelling the grid integration of power to gas: A case study of Denmark," Master's thesis, Chalmers University of Technology, Sweden, 2014.

[10] A. Martinez-Mares and C. R. Fuerte-Esquivel, "A unified gas and power flow analysis in natural gas and electricity coupled networks," IEEE Transactions on Power Systems, vol. 27, no. 4, pp. 2156-2166, Nov. 2012.

[11] M. Shahidehpour, Y. Fu, and T. Wiedman, "Impact of natural gas infrastructure on electric power systems," IEEE Proceedings, vol. 93, no. 5, pp. 1042-1056, May 2005

[12] A. Quelhas, E. Gil, J. D. McCalley, and S. M. Ryan, "A multiperiod generalized network flow model of the U.S. integrated energy system: Part I-Model description," IEEE Transactions on Powver Systems, vol. 22, no. 2, pp. 829-836, May 2007.

[13] X. Zhang, M. Shahidehpour, A. S. Alabdulwahab, and A. Abusorrah, "Security-constrained cooptimization planning of electricity and natural gas transportation infrastructures," IEEE Transactions on Power Systems, vol. 30, no. 6, pp. 2984-2993, Nov. 2015. 
[14] X. Xu, Hongjie Jia, H.-D. Chiang, D. C. Yu, and D. Wang, "Dynamic modeling and interaction of hybrid natural gas and electricity supply system in microgrid," IEEE Transactions on Power Systems, vol. 30, no. 3, pp. 1212-1221, May 2015.

[15] Q. Zeng, J. Fang, J. Li, and Z. Chen, "Steady-state analysis of the integrated natural gas and electric power system with bi-directional energy conversion," Applied Energy, vol. 184, pp. 1483-1492, 2016.

[16] S. Clegg and P. Mancarella, "Storing renewables in the gas network: Modelling of power-to-gas seasonal storage flexibility in low-carbon power systems," IET Gener. Transm. Distrib, vol. 10, no. 3, pp. 566-575, 2016.

[17] S. Clegg and P. Mancarella, "Integrated modeling and assessment of the operational impact of powerto-gas (P2G) on electrical and gas transmission networks," IEEE Trans. Sustain. Energy, vol. 6, no. 4, pp. 1234-1244, 2015.

[18] H. Khani and E. Z. Farag, "Optimal day-ahead scheduling of power-to-gas energy storage and gas load management in wholesale electricity and gas markets," IEEE Trans. Sustain. Energy, vol. 9, no. 2, pp. 940-951, 2018

[19] L. Bai, F. Li, H. Cui, T. Jiang, H. Sun, and J. Zhu, "Interval optimization based operating strategy for gas-electricity integrated energy systems considering demand response and wind uncertainty," Applied Energy, vol. 167, pp. 270-279, 2016.

[20] S. An, "Natural and electricity optimal power flow," Ph.D. thesis, Oklahoma State University, May 2004.

[21] Mathwork Inc., MATLAB Optimization Toolbox. Available: https://www.mathworks.com/ help/pdf_doc/optim/optim_tb.pdf

[22] National Grid Electricity Transmission, Plc., The Grid Code Issue 4, Revision 11. UK: National Grid Electricity Transmission, Plc., March 16th, 2012. 